veteran-nonveteran mortality risk, strategies for addressing data limitations, and directions for future research.

\section{AGING VETERANS AND LONG-TERM OUTCOMES OF MILITARY SERVICE: IMPLICATIONS FOR PRACTICE AND POLICY}

R.A. Settersten, Jr., Oregon State University, Corvallis, Oregon, United States

This paper builds on the scientific output of the NIAfunded Research Network on Lifespan Outcomes of Military Service (R24-AG039343), which was formed to advance interdisciplinary study of the long-term effects of service on later-life health and well-being. The Network generated an inventory of existing longitudinal studies and variables relevant to military service, created a website to disseminate this information, and mined these datasets for collaborative research. This presentation considers the implications of the Network's evidence for practice and policy meant to improve the welfare of veterans and their families. These include specific strategies for fostering resilience, strengthening social relationships and networks, promoting physical and mental health, and designing interventions at specific points of transition in military life. It also offers strategies for better gauging the needs of aging veterans, increasing workforce capacity to serve them, and building data resources and conducting analyses to improve veterans' experiences in healthcare systems.

\section{SESSION 1340 (SYMPOSIUM)}

\section{CHILDHOOD RISK AND PROTECTION FOR ADULT COGNITION}

Chair: E. Greenfield, Rutgers, The State University of New Jersey, New Brunswick, New Jersey

Co-Chair: S. Moorman, Boston College, Chestnut Hill, Massachusetts

Discussant: C.A. Reynolds, University of California

Riverside, Riverside, California

Drawing on insights from the life course perspective, this symposium examines how social inequalities originating in childhood have implications for cognitive aging decades later. The papers draw from four large, longitudinal population studies that have repeated measures of adult cognitive function: the China Health and Retirement Longitudinal Study (CHARLS), the National Survey of Midlife in the U.S. (MIDUS), the Wisconsin Longitudinal Study (WLS), and the Vietnam Era Twin Study of Aging (VETSA). The first two papers focus on personal relationships in childhood. Using data from CHARLS, the first paper provides evidence that better quality relationships with friends and parents in childhood is a robust predictor of cognition in middle and later life. The second paper, using MIDUS, finds that linkages between parenting practices and midlife cognition differ for low-SES versus high-SES families. The second two papers address the confluence of risk and protective factors across ecological domains in childhood. Using data from VETSA, the third paper demonstrates an interaction between rural residence and childhood socioeconomic status (SES), suggesting that the availability of resources (based on geographic context) and accessibility of resources (based on family SES) in childhood jointly influence later life cognition. The fourth paper uses a latent class approach with data from the WLS and finds that clusters of advantage/disadvantage across geographic setting, school quality, and family SES are associated with memory and language/ executive functioning at age 65 . Together, these papers suggest the importance of better specifying childhood risk and protection within life course epidemiological research on cognitive aging.

\section{THE INFLUENCE OF EARLY-LIFE SOCIAL RELATIONSHIPS ON COGNITIVE FUNCTION} AMONG OLDER ADULTS IN CHINA

Z. Zhang ${ }^{1}$, H. Xu, $\mathrm{PhD}^{2}$, L. Li, $\mathrm{PhD}^{3}$, J. Liu, $\mathrm{PhD}^{4}, 1$. Michigan State University, Lansing, Michigan, United

States, 2. University of Michigan, Ann Arbor, MI, USA., 3. University of Michigan, Ann Arbor, MI, USA. , 4. Columbia University, New York, NY, USA.

Previous research has shown that childhood conditions have long-term effects on cognitive function in later life. We contribute to this literature by investigating the effects of early-life social relationships on cognitive function and cognitive change among adults aged 50 and older in China. We used data from the China Health and Retirement Longitudinal Study (CHARLS), 2011-2013, and its supplement - the 2014 Life History Survey. Our analytic sample included 11,170 respondents. Cognitive function was measured by episodic memory and mental intactness. Our results show that having good friends and positive parent-child relationships in early life were associated with higher cognitive function at baseline and slower cognitive decline during the 2-year follow-up. Educational attainment accounted for some, but not all, of the associations. Our findings suggest that positive social relationships in early life may have longterm beneficial effects on cognitive function in later life.

\section{SOCIOECONOMIC STATUS AND PARENTING STYLE IN CHILDHOOD: LONG-TERM EFFECTS ON COGNITION AT MIDLIFE}

Y. Liu ${ }^{1}$, M.E. Lachman, $\mathrm{PhD}^{2}$, 1. Brandeis University, Dartmouth, Massachusetts, United States, 2. The Gerontological Society of America, Washington, DC, Brandeis University, Waltham, MA, USA

The study assessed whether childhood socioeconomic status (SES) is related to cognitive function and cognitive change at midlife and explores the buffering effect of adulthood SES and parenting style. Data were derived from the three waves of the Midlife in the United States (MIDUS) study, a national survey including 7,108 participants. Multilevel modeling results revealed that adulthood SES moderated the effect of childhood SES on cognitive function. Interactions showed that paternal discipline was positively related to cognitive function among participants with low childhood SES, and negatively related to cognitive function among participants with high childhood SES. Paternal and maternal affection were positively related to cognitive function among participants with high childhood SES, and negatively related to cognitive function among participants with low childhood SES. The findings advance the understanding of the long-term consequences of childhood adversity and other early life experiences for cognitive functioning in midlife. 